\title{
Surface functionalization of SPR chip for specific molecular interaction analysis under flow condition
}

\author{
Tao Ma*, Xiaoxia Chen ${ }^{\dagger}$, Qing Peng ${ }^{\star}, \|$, Pengfei Zhang \\ and Yonghong $\mathrm{He}^{\S, \uparrow}$ \\ *Department of Ophthalmology, Shanxi Eye Hospital \\ Taiyuan 030002, P. R. China \\ ${ }^{\dagger}$ Department of Ophthalmology \\ Xin Hua hospital affiliated to Shanghai \\ JiaoTong University School of Medicine \\ Shanghai 200092, P. R. China \\ ¿Department of Ophthalmology, MinHang Hospital \\ Affiliated to Fudan University \\ Shanghai 201199, P. R. China \\ ${ }^{\S}$ Shenzhen Key Laboratory for Minimal Invasive Medical Technologies \\ Institute of optical imaging and sensing \\ Graduate School at Shenzhen \\ Tsinghua University, Shenzhen 518055, P. R. China \\ "Department of Physics, Tsinghua University \\ Beijing 100084, P. R. China \\ "lfudanpengqing@sina.com
}

Received 29 December 2015

Accepted 4 May 2016

Published 9 June 2016

\begin{abstract}
Surface functionalization of sensor chip for probe immobilization is crucial for the biosensing applications of surface plasmon resonance (SPR) sensors. In this paper, we report a method circulating the dopamine aqueous solution to coat polydopamine film on sensing surface for surface functionalization of SPR chip. The polydopamine film with available thickness can be easily prepared by controlling the circulation time and the biorecognition elements can be immobilized on the polydopamine film for specific molecular interaction analysis. These operations are all performed under flow condition in the fluidic system, and have the advantages of easy implementation, less time consuming, and low cost, because the reagents and devices used in the operations are routinely applied in most laboratories. In this study, the specific absorption
\end{abstract}

$\|_{\text {Corresponding author. }}$

This is an Open Access article published by World Scientific Publishing Company. It is distributed under the terms of the Creative Commons Attribution 4.0 (CC-BY) License. Further distribution of this work is permitted, provided the original work is properly cited. 
between the protein A probe immobilized on the sensing surface and human immunoglobulin $\mathrm{G}$ in the buffer is monitored based on this surface functionalization strategy to demonstrated its feasibility for SPR biosensing applications.

Keywords: Surface plasmon resonance; surface functionalization; dopamine; molecular interaction analysis.

\section{Introduction}

Surface plasmon resonance (SPR) sensors play important roles in specific molecular interaction analysis owing to the features of high sensitivity, label free, and real-time monitoring. ${ }^{1}$ The SPR biosensing platforms are usually combined with the fluidic system for sample transfer to the sensing surface, and with the immobilized biorecognition elements for specific measurements of chemical and biological substances. ${ }^{2}$ The detailed information on kinetics of the molecular interaction can be achieved by SPR biosensors to facilitate measurements accurately without complicated treatments for specimen using reporter elements. ${ }^{3}$

The SPR sensors usually employ the evanescent field generated by the free electron oscillation in the gold film for measurements. ${ }^{4}$ The biomolecular owning hydrophilic property cannot be absorbed by the bare metallic film owning hydrophobic property, so the surface of SPR chip requires to be modified for probe immobilization. The gold surface is usually functionalized with mixed alkanethiols. The probes are fixed by the functional groups, such as surface-exposed amino group and activated carboxyl group, which are attached to the gold surface via gold-sulfhydryl linkage. ${ }^{2}$ This method can be operated by immersing SPR chips in the reagents, ${ }^{5-7}$ or circulating the reagents serially through the sensing surface. $^{8}$

Recently, the polydopamine film, which is a great binding media for surface modification on the inorganic and organic surfaces, ${ }^{9}$ is pointed to be an economical and convenient solution for rapid surface functionalization. ${ }^{10}$ The reported surface modification method for label free biosensors is usually operated by immersing the sensor chip in the dopamine aqueous solution to coat the polydopamine film on the sensing surface using dopamine self-polymerization. ${ }^{11,12}$ The polydopamine films are not only coated on the sensing surface of SPR chip but also the other surface due to good adhesion of dopamine, and the polydopamine film on the surfaces of SPR chips apart from the sensing surface should be removed to avoid the adverse effect on measurement before it is attached to the experimental system. ${ }^{13}$ But the dopamine is sensitive to the light and air, ${ }^{14}$ so the removing process of polydopamine film, which inevitably makes the modification films exposed to the environment, has negative effect on the quality of surface modification and complicates the operations.

In this paper, we describe an improved surface functionalization approach using polydopamine film for SPR biosensors, where the polydopamine film is prepared easily by circulating the dopamine aqueous solution through the sensing surface. The realtime measurement using the SPR sensor shows that the polydopamine film with different thicknesses can be achieved by controlling the circulation time. The surface modification and specific measurement can be operated in the same fluidic systems, so the polydopamine film will not be exposed to the environments and can be prepared with simple operations. The polydopamine film coated within about 10 min is prepared for the biochemical experiment, and the sensing surface coated with polydopamine film is investigated using the scanning electric microscope (SEM). Then, the specific absorption between the protein A probe immobilized on the sensing surface and human immunoglobulin $\mathrm{G}$ (IgG) in the buffer is measured to demonstrate the feasibility of proposed method for the surface functionalization of SPR biosensors.

\section{Materials and Instrumentation}

\subsection{Reagents}

$\mathrm{KH}_{2} \mathrm{PO}_{4}, \mathrm{Na}_{2} \mathrm{HPO}_{4}, \mathrm{KCl}, \mathrm{NaCl}, \mathrm{HCl}$, bovine serum albumin (BSA), Tris, dopamine, and glucose are achieved from Alfa-Aesar (USA), in molecular biology grade or higher. Human IgG and protein A are achieved from Tianze Biotechnology (China). The Milli-Q system is employed in this study to 
acquire the deionized water. The composition of PBS buffer is $1.4 \mathrm{mM} \mathrm{KH}_{2} \mathrm{PO}_{4}, 2.7 \mathrm{mM} \mathrm{KCl}, 8 \mathrm{mM}$ $\mathrm{Na}_{2} \mathrm{HPO}_{4}, 137 \mathrm{mM} \mathrm{NaCl} .500 \mu \mathrm{g} / \mathrm{mL} \mathrm{BSA}$ is added to the PBS for $\mathrm{PBS}_{\mathrm{BSA}}$ buffer preparation. The Tris buffer is composed of $10 \mathrm{mM}$ Tris with the $\mathrm{pH}$ of 8.5 , which is adjusted using $\mathrm{HCl} .2 \mathrm{mg} / \mathrm{mL}$ dopamine is added to the Tris buffer for dopamine aqueous solution preparation.

\section{2. $\quad$ SPR sensing system setup}

The polished SF4 optical glass substrates are ultrasonically cleaned using the solution containing ethyl alcohol and acetone in the ratio of 1:1. Then the deionized water is used to rinse the substrates, and nitrogen gas flow is applied to dry the substrates. Then, a $2 \mathrm{~nm}$ chromium film as adhesive layer and a $50 \mathrm{~nm}$ gold film as surface plasmon active layer are serially coated on these substrates by magnetron sputtering to construct SPR sensor chips. In the film deposition process, the substrates are heated to $350^{\circ} \mathrm{C}$ and applied a negative voltage of $150 \mathrm{~V}$ for the film quality improvement. ${ }^{15}$ The film thickness and deposition rate are measured by an oscillator. Finally, the SPR chips are attached to SF4 prisms utilizing RI matching oil (Cargille) for construction of SPR modules.

A common one-dimensional SPR imaging system based on angle interrogation combined with a temperature stabilization system is applied as shown in Fig. 1(a). The light source (LS) is constructed by a $10 \times$ objective lens, a light emitting diode (LED, Osram W5AP, Germany, center wavelength

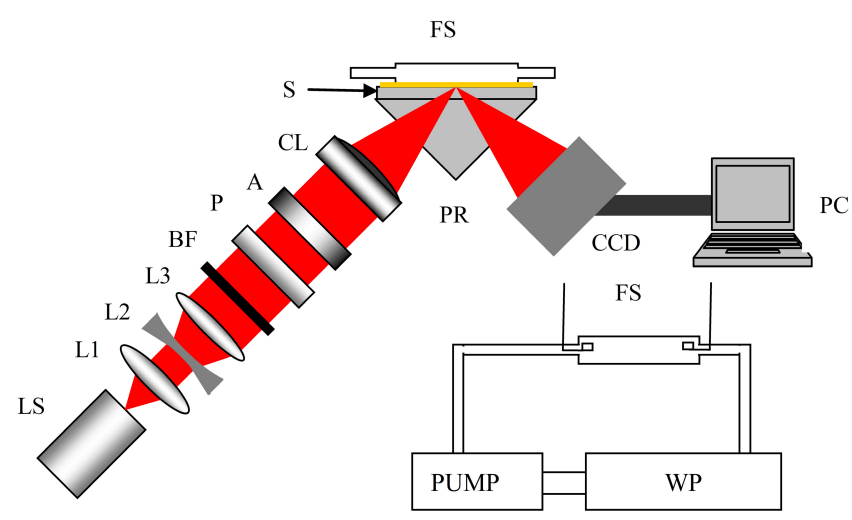

(a)
$632.8 \mathrm{~nm}$ ) and a pinhole. The objective lens focuses the light emitted by the LED to the pinhole. Then a collimation lens group described in Ref. 16 is applied to acquire the collimated light beam with high quality. This lens group is constructed by two convex lenses (L1, L3) and one concave lens (L2). Then the collimated light beam is filtered using a bandpass filter (BF, FL632.8-10, Thorlabs), and then transverse magnetic polarized using the polarizer $(\mathrm{P})$. Aperture (A) is employed in this system for a rectangular light spot. Then the cylindrical lens ( $f=50 \mathrm{~mm}$, Thorlabs) are used to focus the incident light to a narrow line on the surface of SPR chip to constitute the imaging channel. The SPR sensor module is fixed as the Kretschmann configuration. A plexiglass flow system (FS) with two fluidic channels $(10 \mathrm{~mm} \times 2 \mathrm{~mm} \times 2 \mathrm{~mm}, 40 \mu \mathrm{l})$ as shown in Fig. 1(b) is attached to the SPR module for sample transfer. In the FS, two fluidic channels play the roles of detection, and reference channels, respectively. The reflection lights are captured using a charge coupled device $(2580 \times 1912$ pixels, $3.4 \mu \mathrm{m} \times 3.4 \mu \mathrm{m}$ pixel size, ICX282AQ, Sony) for angular spectral analysis to achieve the resonance angle information using the personal computer (PC). The temperature control system is configured in the manner described in Ref. 15. A multichannel peristaltic pump (PUMP) is employed to circulate the sample through the programmable water bath (WP) and FS.

In this experimental system, the facula illuminating the cylindrical lens has the size of $10 \mathrm{~mm}^{2} \times 10 \mathrm{~mm}^{2}$. Considering the focus length of $50 \mathrm{~mm}$ and prism refraction effect, the incident angular range is

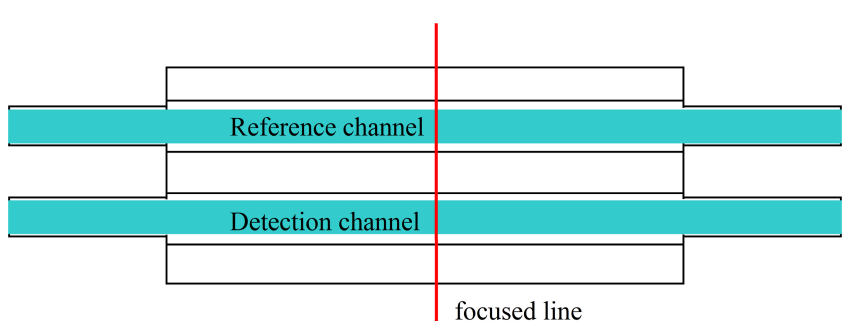

(b)

Fig. 1. (a) Schematic of experimental system combined with the temperature stabilization: LS, light source; L1, L3, convex lens; L2, concave lens; BF, bandpass filter; P, linear glass polarizer; A, rectangular aperture; CL, cylindrical lens; PR, prism; S, SPR chip; FS, fluidic system; CCD, charge coupled device; PC, personal computer. Inset: Schematic representation of temperature control system; WP, water bath; PUMP, peristaltic pump. (b) Schematic of dual channel fluidic system. 
approximately $6.52^{\circ} \mathrm{C}$. The reflectance light recorded by the CCD owns the size of approximately $20 \mathrm{~mm}^{1} \times 10 \mathrm{~mm}^{2}$. Using the pixel size, the system angular resolution can be estimated to be $1.1 \times 10^{-3}{ }^{\circ} \mathrm{C}$ pixel.

\section{Results and Discussions}

\subsection{Refractive index test}

The deionized water and glucose solutions with concentrations from $1 \mathrm{~g} / \mathrm{L}$ to $5 \mathrm{~g} / \mathrm{L}$ and the increasement of $1 \mathrm{~g} / \mathrm{L}$ are detected sequentially to test the system performance. The sample temperature is configured at $30^{\circ} \mathrm{C}$. The water, whose refractive index (RI) is not affected by evaporation, is used to create the baseline. The solution RI raises $1.515 \times$ $10^{-4}$ refractive index unit (RIU) with the concentration increasement of $1 \mathrm{~g} / \mathrm{L}$. 50 images are captured for measurements of each sample and the exposure time is set at $10 \mathrm{~ms}$.

The measured resonance angles as the function of corresponding image numbers are shown in Fig. 2(a). It can be seen that the resonance angle shifts to the pixel position with small value when glucose concentration arises. Due to the system characteristics, the zone in the CCD sensing surface, where the pixel numbers in the captured image are represented by small values, receives the reflected light with large incident angle. Considering these, the phenomenon indicates the resonance angle shifts to larger value when the glucose concentration rises, which agrees well with the reported results. ${ }^{15,16}$ The averaged measured resonance angle is plotted against sample RI and shown in Fig. 2(b). It can be seen that this system responds linearly for the RI, and the slope can be calculated to achieve the sensitivity factor $(\mathrm{SF})$ of $6.6 \times 10^{4}$ pixels/RIU. The RI resolution $\left(\sigma_{\mathrm{RI}}\right)$ can be achieved based on the baseline standard deviation $\left(\sigma_{\mathrm{SO}}\right)$ and $\mathrm{SF}$ on the basis of

$$
\sigma_{\mathrm{RI}}=\frac{\sigma_{\mathrm{SO}}}{\mathrm{SF}}, \quad \mathrm{SF}=\frac{\partial \theta}{\partial n},
$$

where the $\theta$ is the incident angle, $n$ is sample RI. ${ }^{17}$ Using the baseline achieved by deionized water and the system angular resolution, the SF in degree and $\mathrm{RI}$ resolution can be calculated to be $72.6^{\circ} \mathrm{C}$ and $1.3 \times 10^{-5} \mathrm{RIU}$, which are at the same level with current SPR biosensors. ${ }^{2}$ This indicates that this system can be used for the biochemical applications.

\subsection{Surface functionalization with polydopamine film}

The dopamine aqueous solutions are circulating through the sensing surface of SPR chip in the fluidic system, and the temperature is configured at $30^{\circ} \mathrm{C}$. The SPR sensor responses to dopamine selfpolymerization on the surface of SPR chip, namely the polydopamine film coating, in the reference, and detection channels are recorded in real time and shown in Figs. 3(a) and 3(b). The dopamine solution is circulating for $10 \mathrm{~min}$, and then the PBS buffer is used to wash away uncombined dopamine molecules. The time interval is $10 \mathrm{~s}$ and the exposure time is fixed at $10 \mathrm{~ms}$ for these images acquisition. It can be seen that the rate of resonance angle shift slows down with time. This should be because the formation of the polydopamine film contains the absorption and desorption effects of the dopamine molecules, and the interaction between

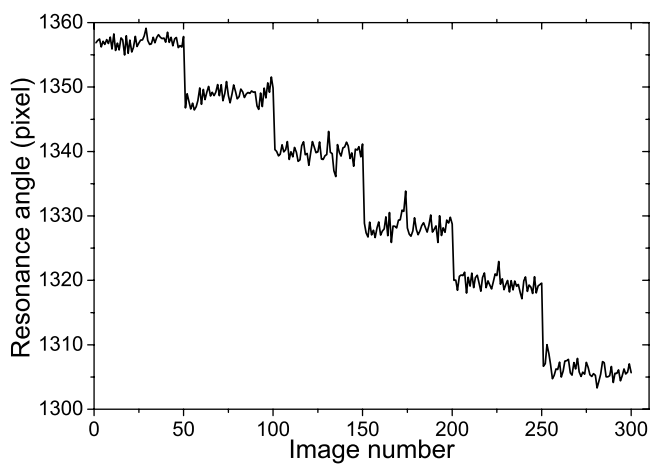

(a)

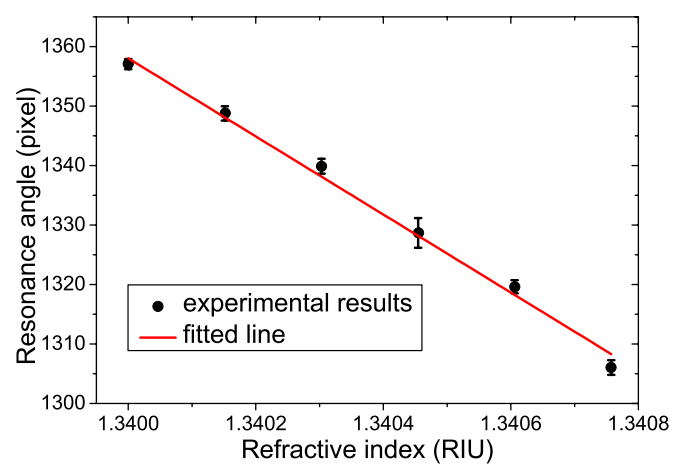

(b)

Fig. 2. (a) The measured resonance angle as the function of corresponding captured image numbers. (b) The averaged measured resonance angle plotted against the refractive index. The error bars represent the standard deviations. 


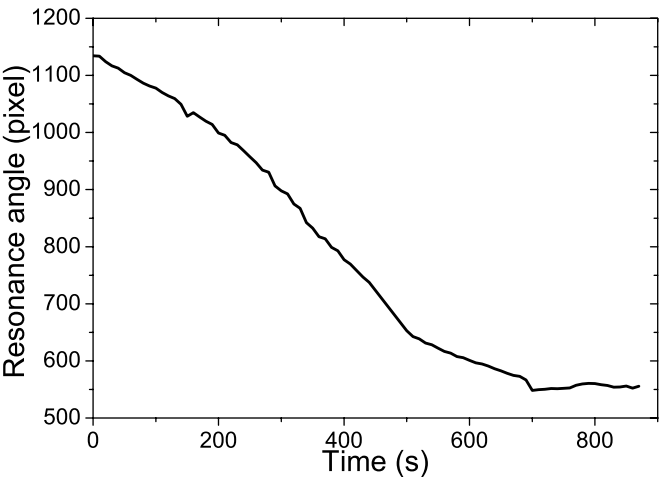

(a)

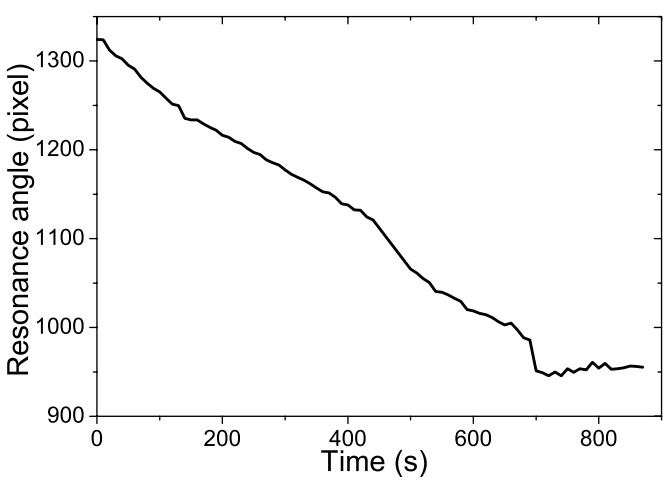

(b)

Fig. 3. (a), (b) The measured resonance angles in reference, detection channels as the function of time for polydopamine film coating.

absorption and desorption processes may be stronger with the increase of absorbed dopamine molecules, which will lead to irregular coating rate of polydopamine film.

The thickness of polydopamine film prepared in this experiment can be estimated using the comparison between the experimental reflectance spectra and the theoretical reflectance spectra calculated by the theory about the surface sensitivity of SPR sensors described in Ref. 18. The experimental reflectance spectra with and without the polydopamine film in the reference channel are shown in Fig. 4(a) and those in the detection channel are shown in Fig. 4(b). It can be seen from Figs. 4(a) and 4(b) that the resonance angle shifts caused by the polydopamine film in the reference, and detection channel are approximately 600 and 400 pixels, which represent $0.66^{\circ} \mathrm{C}$ and $0.44^{\circ} \mathrm{C}$ in this system. These resonance angle shifts basically agree with the theoretical predictions when the SRP sensor is coated with polydopamine film with the thickness of $4 \mathrm{~nm}$ and $3 \mathrm{~nm}$, whose reflectance spectra are shown in Figs. 4(c) and 4(d), respectively. The thickness difference between the polydopamine films may be caused by the different surface characteristics caused by the film uniformity and different flow conditions in different channels. The evanescent wave penetration depth of SPR sensor is usually at the level of $400 \mathrm{~nm},{ }^{15}$ so the polydopamine film with the thickness of several nanometers cannot affect the SPR sensor characteristics. Using the electromagnetic theory described in Ref. 18, the SFs in degree for the SPR sensors without polydopamine film, with $3 \mathrm{~nm}$ and $4 \mathrm{~nm}$ polydopamine films are $72.3^{\circ} \mathrm{C}, 72.2^{\circ} \mathrm{C}$, and $72.2^{\circ} \mathrm{C}$. The differences among these SFs are very small and can be neglected in the practical applications. The SPR sensors with 10, 20, 30, 40 and $50 \mathrm{~nm}$ polydopamine films have SFs of $72.0^{\circ} \mathrm{C}$, $71.2^{\circ} \mathrm{C}, 68.6^{\circ} \mathrm{C}, 64.7^{\circ} \mathrm{C}$, and $59.7^{\circ} \mathrm{C}$. This indicates that the SPR sensor's sensitivity has obvious variation when the polydopamine film thickness is larger than $20 \mathrm{~nm}$.

To intuitively observe the polydopamine film on the sensor surface, the SPR chips without and with the polydopamine films are observed using the SEM (Nova Nano SEM 230). The SPR chip without polydopamine film is observed using electron accelerate voltages of $3000 \mathrm{~V}$, and $15000 \mathrm{~V}$ as shown in Figs. 5(a) and 5(a), respectively. The SPR chip with the polydopamine film is prepared by immersing the chip in the dopamine solution, which is placed in the shaking table to simulate the flow condition and observed using electron accelerate voltages of $15000 \mathrm{~V}$ as shown in Fig. 5(c). Considering the polydopamine film has very thin thickness, the electron acceleration voltage is adjusted to a high value of $15000 \mathrm{~V}$ to achieve higher resolution for clear observation of polydopamine film. The SPR chip without polydopamine film is observed using accelerate voltage of $15000 \mathrm{~V}$ for comparison. The gold film coating using magnetron sputtering is based on island growth dynamic process, ${ }^{15}$ so the gold atoms located in the junction of different islands are not tightly adhesive to the $\mathrm{Cr}$ layer and they could be blown away by high-energy electrons, which lead to the black lines in the micrographs shown in Figs. 5(b) and 5(c). When the electron accelerate voltage is adjusted to be a lower value, the surface topography of the gold film prepared by magnetron sputtering can be observed more closely 


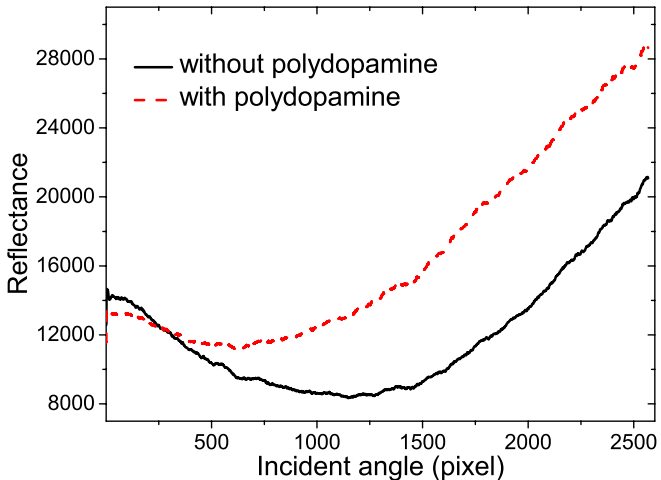

(a)

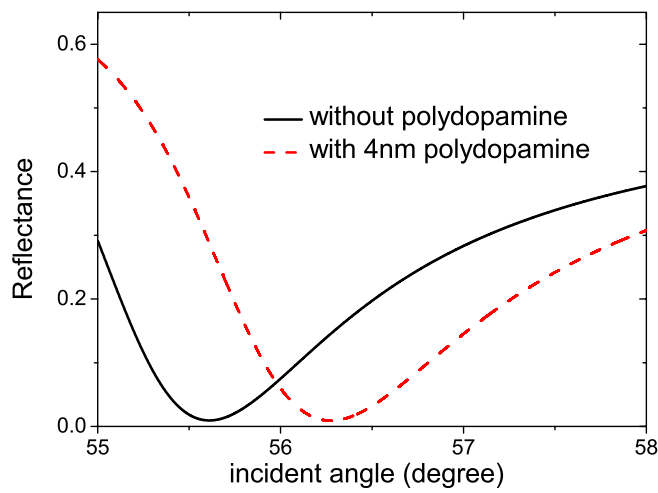

(c)

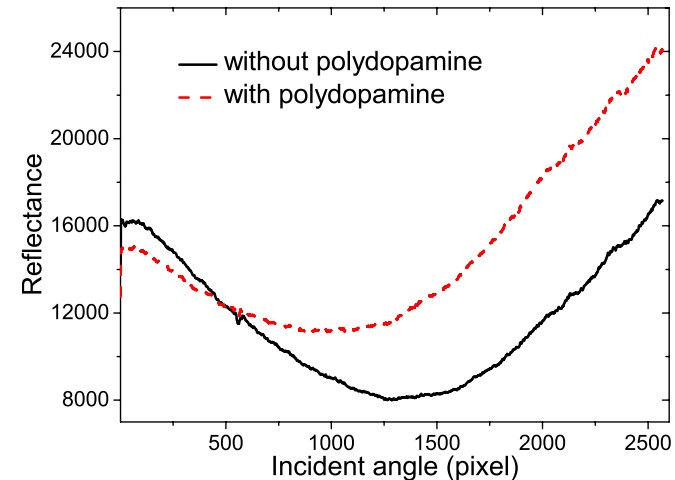

(b)

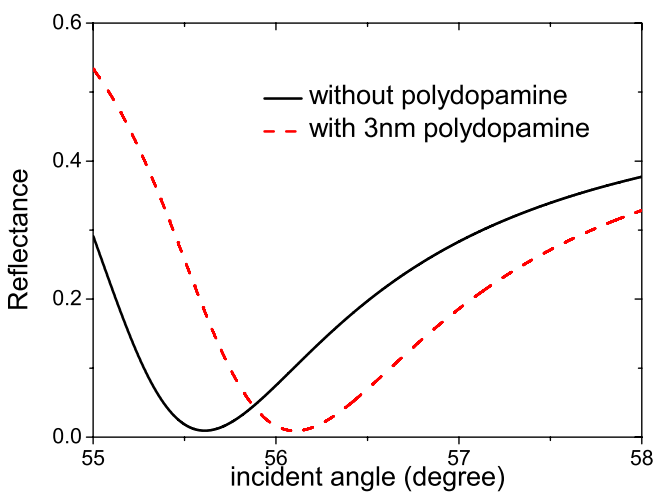

(d)

Fig. 4. (a) The measured reflectance spectra without and with polydopamine in the reference channel. (b) The measured reflectance spectra without and with polydopamine in the detection channel. (c) The SPR calculated reflectance spectra without and with $4 \mathrm{~nm}$ polydopamine film. (d) The SPR calculated reflectance spectra without and with $3 \mathrm{~nm}$ polydopamine film.

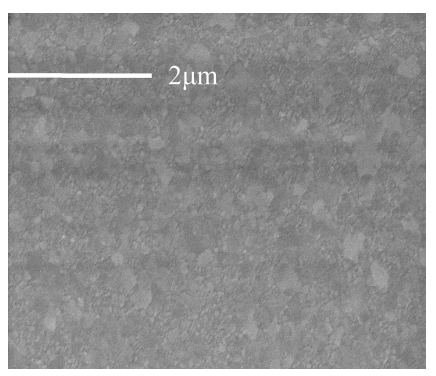

(a)

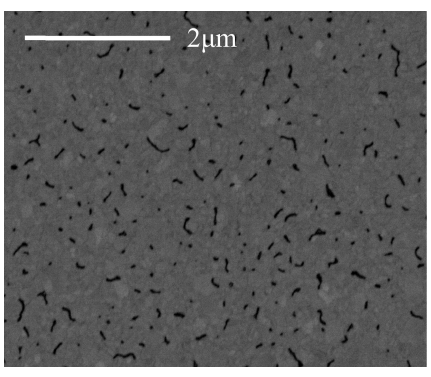

(b)

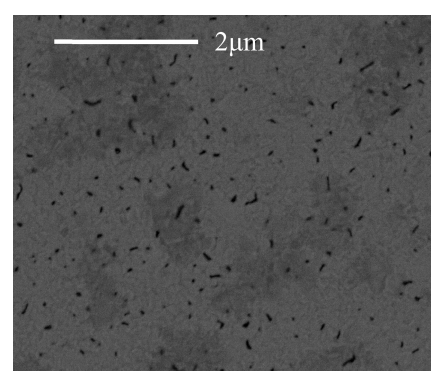

(c)

Fig. 5. (a) Micrograph of SPR chip with electron acceleration voltage of 3000 V. (b) Micrograph of SPR chip with electron acceleration voltage of $15000 \mathrm{~V}$. (b) Micrograph of SPR chip with the polydopamine film with electron acceleration voltage of $15000 \mathrm{~V}$.

to the actual situations as shown in Fig. 5(a). The high-energy electrons also may blow away some dopamine polymers, which results in the uncontinuous polydopamine film as shown in Fig. 5(c). Despite these, it can be obviously seen from Fig. 5 that there are agglomerates on the surface of SPR chip with polydopamine film. This demonstrates the feasibility of surface polydopamine functionalization under flow condition via the self-polymerization of dopamine molecules. 


\subsection{Biomolecular interaction analysis}

The specific absorption between the protein A probe immobilized on the sensing surface and human IgG in the buffer is measured by this system to demonstrate that this surface functionalization method can be applied for biomolecular interaction analysis. The protein $\mathrm{A}$ and $\operatorname{IgG}$ aqueous solutions are prepared by adding $100 \mu \mathrm{g} / \mathrm{mL}$ protein $\mathrm{A}$ and IgG to PBS buffers, respectively. First, the protein A solution is circulated in detection channel to create a protein $\mathrm{A}$ biorecognition layer on the sensing surface. The measured resonance angle of the detection channel is plotted against time as shown in Fig. 6(a). Then the $\mathrm{PBS}_{\mathrm{BSA}}$ buffer is circulated in both channels to block the bare polydopamine film surface using BSA. The measured resonance angles of both channels are plotted against time as shown in Fig. 6(b). It can be seen that the protein molecules are fixed on the polydopamine rapidly. After these operations, the surface chemical state of SPR chip is prepared for the specific biomolecular interaction analysis. The polydopamine film can be attached to the surfaces of diverse kinds of materials. This is because the catechol groups in the dopamine molecules are oxidized to form the dopamine quinine, and then the crosslinked composite layer between the polydopamine film and material surface is produced via the disproportionation reaction between the dopamine and dopamine quinine. ${ }^{19}$ The polydopamine film contains a variety of functional groups, such as the adjacent hydroxide groups and quinine groups. The quinine groups can react with the nucleophiles, which provide conditions for the biofunctionalization of the polydopamine film surface. The conjugation between the protein molecules and polydopamine film can be owing to the Michael addition reaction and Schiff base reaction. ${ }^{20}$ In the practical applications, the surface modification method using polydopamine film is usually limited by the fact that the dopamine molecules are not only self-polymerized on the sensor surface but also in the bulk solution. To suppress the useless self-polymerization effect, complicated operations such as photophobic and nonoxidation technical process are employed in our previous work. ${ }^{21,22}$ Although the surface functionalization method proposed in this paper simplify the operation process and can be realized easily by circulating the dopamine solution through the sensor surface, the operation time also should be as short as possible.
The IgG solution is circulated in both channels and then the $\mathrm{PBS}_{\mathrm{BSA}}$ buffer begins to be circulated in both channels at $3000 \mathrm{~s}$. The measured resonance angles as the functions of time for both channels are shown in Fig. 6(c). It can be seen that the specific absorption between the IgG in the sample and the immobilized protein $\mathrm{A}$ can be observed in the detection channel, while the resonance angle shift in the reference channel should be caused by the nonspecific absorption. To show the measurement result of specific absorption between IgG and protein A clearly, the referenced measurement resonance angle, namely the different values between the measurement resonance angles of the reference and detection channels, as function of time is shown in Fig. 6(d). It can be seen that the absorption speed decreases with absorption time, which should be because the specific molecular interaction contains both absorption and diffusion effects, and the diffusion effect is more distinct when the immobilized protein $\mathrm{A}$ absorption centers on the sensing surface are fewer. It should also be noticed in Figs. 6(c) and 6 (d) that the resonance angles do not have obvious shifts when the $\mathrm{PBS}_{\mathrm{BSA}}$ buffer is pumped into the fluidic channels instead of the IgG solutions, which indicates that the specific binding between the immobilized protein A and IgG is firm. This experiment shows that the SPR system combined with the polydopamine film prepared by the proposed surface functionalization method can be used for specific biomolecular interaction analysis. To achieve the upper detection limits of the SPR biosensors proposed in this paper, IgG solutions with different concentrations are measured in this experiment. The $30 \mu \mathrm{g} / \mathrm{mL}$ and $50 \mu \mathrm{g} / \mathrm{mL}$ IgG solutions are measured as shown in Fig. 6(e). It can be seen that the resonance angle shifts measuring 30 $\mu \mathrm{g} / \mathrm{mL}$ and $50 \mu \mathrm{g} / \mathrm{mL}$ IgG solutions are about 250 and 400 pixels, which indicate that the resonance angle shift responds linearly to the protein A concentration. Considering that the resonance angle shift measuring $100 \mu \mathrm{g} / \mathrm{mL}$ IgG solution is about 600 pixels, the saturation concentration measuring the IgG solution using protein A probes immobilized on the sensor surface should be about $75 \mu \mathrm{g} /$ $\mathrm{mL}$ based on the linear correspondence axiom. The $1 \mu \mathrm{g} / \mathrm{mL}$ IgG solutions are measured to achieve the lower detection limit as shown in Fig. 6(f). It can be seen that the process of specific absorption between the IgG and protein A can be recognized although the fluctuations of binding curve measuring 


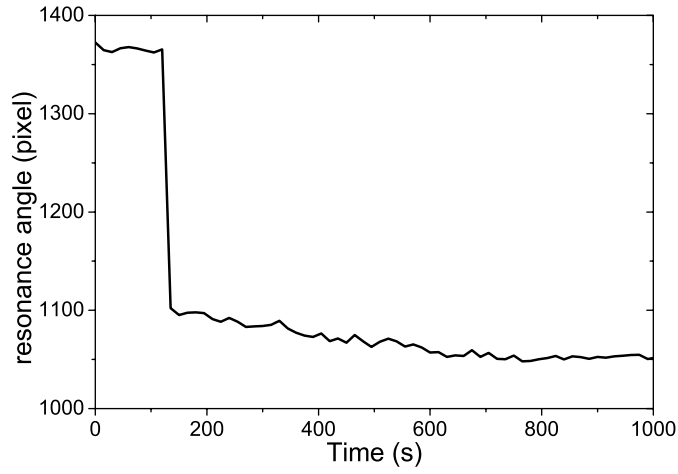

(a)

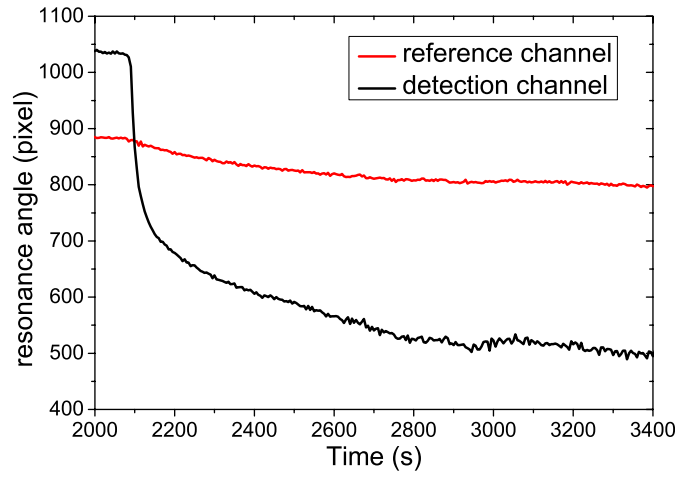

(c)

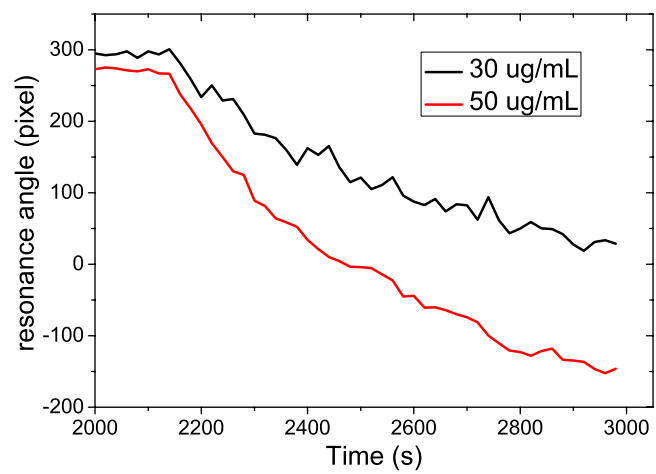

(e)

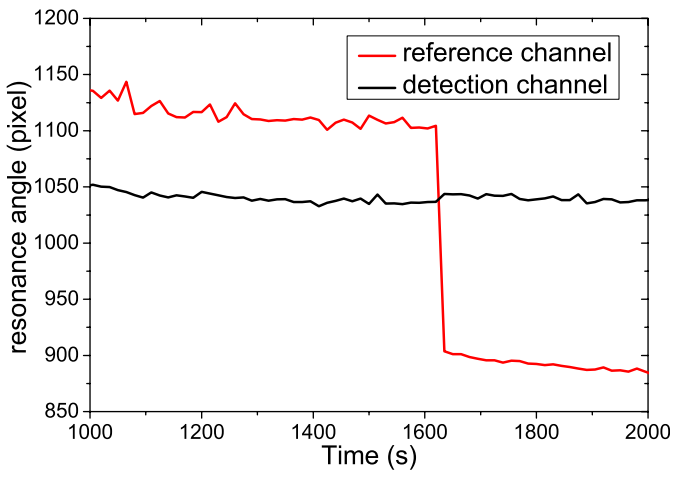

(b)

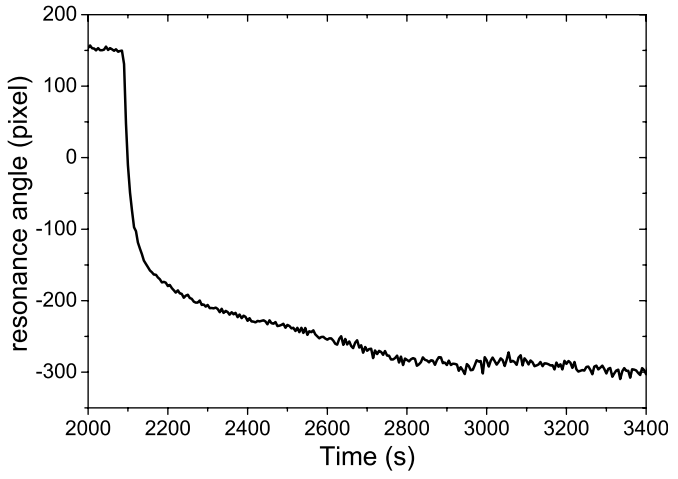

(d)

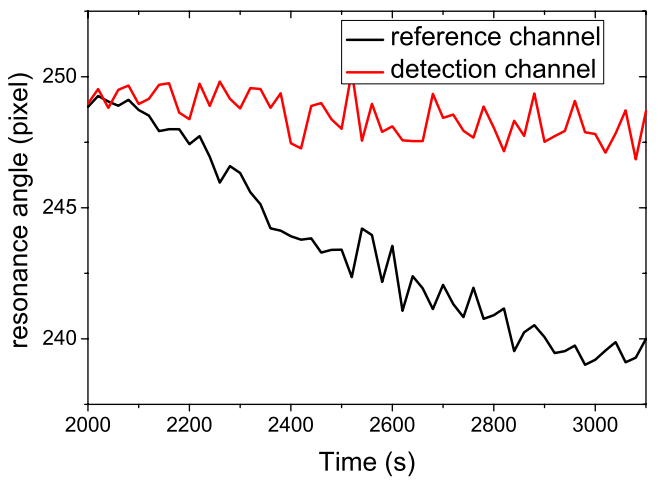

(f)

Fig. 6. (a) The measured resonance angle plotted against time in the detection channel pumping the protein A solution into the

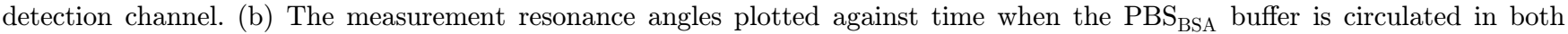
channels. (c) The measurement resonance angles plotted against time in both channels monitoring the specific absorption between immobilized protein A probe and IgG in the buffer. (d) The referenced measurement resonance angles plotted against time monitoring the specific absorption between immobilized protein A probe and IgG in the buffer. (e) The referenced measurement resonance angles plotted against time measuring $30 \mu \mathrm{g} / \mathrm{mL}$ and $50 \mu \mathrm{g} / \mathrm{mL}$ IgG solutions. (f) The measurement resonance angles plotted against time in both channels measuring $1 \mu \mathrm{g} / \mathrm{mL}$ IgG solution.

$1 \mu \mathrm{g} / \mathrm{mL}$ IgG solution are large. So the lower detection limit of this biosensing system is better than the protein analyte concentration of $1 \mu \mathrm{g} / \mathrm{mL}$, which is comparable to the performances of reported SPR biosensors. $^{2}$

\section{Conclusion}

In this paper, we develop a surface modification method using polydopamine film for SPR biosensor. The feasibility of this method for the biochemical application is demonstrated by the antibody-antigen 
interaction analysis using protein A and IgG. The polydopamine film can be prepared easily by circulating the dopamine aqueous solution in the fluidic channels while the desired film thickness can be achieved by controlling the circulation time easily. Besides, considering the dopamine aqueous solution can be prepared with simple operations, we believe that the reported surface modification method provides an economical and convenient choice, which also can be readily realized with commonly used devices in the laboratory and commercial reagents, for the biochemical applications of the SPR biosensors.

\section{Acknowledgments}

This research was made possible with the financial support from NSFC China (61275188, 61378089, $81470029,61361160416)$, the 863 project, China, the Technology Development Program of Shenzhen City, the Committee of Science and Technology Innovation of Shenzhen (JCYJ20140902110354241), and Science and Technology Project of Guangdong Province (2015A010106002).

The first two authors contributed equally to this work.

\section{References}

1. M. A. Cooper, Label-free Biosensors: Techniques and Applications, Cambridge University Press, New York (2009).

2. J. Homola, "Surface plasmon resonance sensors for detection of chemical and biological species," Chem. Rev. 108, 462-492 (2008).

3. R. Karlsson, "SPR for molecular interaction analysis: A review of emerging application areas," J. Mol. Recogn. 17, 151-161 (2004).

4. J. Homola, S. S. Yee, G. Gauglitz. "Surface plasmon resonance sensors: review," Sens. Actuators B, Chem. 54, 3-15 (1999).

5. L. Liu, S. H. Ma, Y. H. Ji, X. Y. Chong, Z. Y. Liu, Y. H. He, H. Ma, "A two-dimensional polarization interferometry based parallel scan angular surface plasmon resonance biosensor," Rev. Sci. Instrum., 82, 023109 (2011).

6. T. Springer, J. Homola, "Biofunctionalized gold nanoparticles for SPR-biosensor-based detection of CEA in blood plasma," Anal. Bioanal. Chem. 404, 2869-2875 (2012).

7. Suherman, K. Morita, T. Kawaguchi, "Effect of alkanethiol molecular structure on sensitivity of surface plasmon resonance sensor," Sens. Actuators B, Chem. 210, 768-775 (2015).

8. P. F. Zhang, L. Liu, Y. H. He, Y. H. Ji, J. Guo, H. Ma, "Temperature-regulated surface plasmon resonance imaging system for bioaffinity sensing," Plasmonics 11, 771-779 (2016).

9. H. Lee, S. M. Dellatore, W. M. Miller, P. B. Messersmith, "Mussel-inspired surface chemistry for multifunctional coatings," Science 318, 426-430 (2007).

10. P. Zhou, Y. Deng, B. Lyu, R. Zhang, H. Zhang, H. Ma, Y. Lyu, S. Wei, "Rapidly-deposited polydopamine coating via high temperature and vigorous stirring, formation, characterization and biofunctional evaluation," Plos One 9, e113087 (2014).

11. H. Shi, Z. Y. Liu, X. X. Wang, J. Guo, L. Liu, L. Luo, J. H. Guo, H. Ma, S. Q. Sun, Y. H. He, "A symmetrical optical waveguide based surface plasmon resonance biosensing system," Sens. Actuators B, Chem. 185, 91-96 (2013).

12. A. Li, Z. Y. Guo, Q. Peng, C. Du, X. Han, L. Liu, J. Guo, Y. H. He, Y. H. Ji, "A saccharides sensor developed by symmetrical optical waveguide-based surface plasmon resonance," J. Innov. Opt. Health Sci., 8, 1550003 (2015).

13. Z. Y. Liu, Q. Peng, H. Shi, S. Q. Sun, J. Guo, X. X. Wang, L. Liu, Y. H. Ji, J. H. Guo, H. Ma, Y. H. He, " $\mathrm{MgF}_{2}-\mathrm{Au}-\mathrm{MgF}_{2}$-polydopamine based surface plasmon resonance sensor and its application in biomedical systems," Analy. Methods, 5, 6306-6311 (2013).

14. S. J. Rego, A. C. Vale, G. M. Luz, J. F. Mano, N. M. Alves, "Adhesive bioactive coatings inspired by sea life," Langmuir 32, 560-568 (2016).

15. P. F. Zhang, L. Liu, Y. H. He, Z. H. Xu, Y. H. Ji, H. Ma, "One-dimensional angular surface plasmon resonance imaging based array thermometer," Sens. Actuators B, Chem. 207, 254-261 (2015).

16. P. F. Zhang, L. Liu, Y. H. He, Z. Y. Shen, J. Guo, Y. H. Ji, H. Ma, "Non-scan and real-time multichannel angular surface plasmon resonance imaging method," Appl. Opt. 53, 6037-6042 (2014).

17. M. Piliarik, J. Homola, "Surface plasmon resonance (SPR) sensors, approaching their limits," Opt. Exp. 17, 16505-16517 (2009).

18. P. F. Zhang, L. Liu, Y. H. He, Y. H. Ji, H. Ma, "Selfreferenced plasmon waveguide resonance sensor using different waveguide modes," J. Sens. 2015, 945908 (2015).

19. M. C. Leeden, "Are conformational changes, induced by osmotic pressure variations, the underlying mechanism of controlling the adhesive activity of mussel adhesive proteins?," Langmuir 21, 1137311379 (2005). 
20. H. Lee, J. Rho, P. B. Messersmith, "Facile conjugation of biomolecules onto surfaces via mussel adhesive protein inspired coating," Adv. Mater. 21, 431-434 (2009).

21. Y. X. Liu, L. Liu, Y. H. He, L. Zhu, H. Ma, "Decoding of quantum dots encoded microbeads using a hyperspectral fluorescence imaging method," Anal. Chem. 87, 5286-5293 (2015).

22. Y. X. Liu, L. Liu, Y. H. He, Q. H. He, H. Ma, "Quantum-dots-encoded-microbeads based molecularly imprinted polymer", Biosens. Bioelectron. 77, 886-893 (2016). 